\title{
COMPARING FEATURE MATCHING ALGORITHMS FOR MEASURING IN-PLANE STRAINS ON CIVIL INFRASTRUCTURES
}

\author{
Youyi Feng ${ }^{1}$, Fei Dai ${ }^{2}$, and Jiaqing Zhao ${ }^{3}$
}

\begin{abstract}
Maintaining structural integrity of civil infrastructures such as bridges and tunnels is always an essential task for civil engineers. Collapse or damage of these infrastructures may lead to a tremendous amount of painful injuries, casualties, and societal losses. This paper reported the work on evaluating optimal feature matching algorithms for development of visual sensing-based techniques to measure in-plane deflections and strains in order to facilitate monitoring and evaluation of integrity of civil infrastructures in a cost-effective way. A series of experiments were conducted in which three algorithms Digital Image Correlation (DIC), Scale-Invariant Feature Transform (SIFT) and Speeded-Up Robust Features (SURF) were compared. The result indicated that DIC has superiority among the three algorithms. To further assess the accuracy of DIC, a high-speed industrial camera was then used to capture a series of continuous image frames of deformed real-world scenarios. The DIC algorithm was adopted in the feature detection and tracking process, and in-plane displacement and strains were calculated and compared with the ground truth. The result indicated that the DIC-based method can achieve highly accurate performance in measuring in-plane deflections and strains for civil infrastructures and holds potential to the development of visual sensing enabled structural health monitoring.
\end{abstract}

Keywords: Image-based methods, structural health monitoring, visual sensing, noncontact measurements, comparative study.

\section{INTRODUCTION}

The aging of constructed civil infrastructures such as bridges, overpasses, dams, and tunnels poses a grand challenge to civil engineers who maintain the integrity and level of service of these structures. A particularly expensive fix is when a structure that has been built does not meet the specifications that were planned. This can be designated as large deflections on the surface material of the structures. Therefore, being able to promptly and effectively measure the deflections or strains of structural members is a crucial element to preserving level of service and structural integrity (Karbhari and Zhao 2000).

Applying visual sensing techniques possesses the potential to tackle this problem by collecting images from distant location and converting the image data into measured distances. The advances of applying visual sensing techniques are allowing practical deployment for large extended systems in a cost-effective way. The image or video data can be used for long-term condition assessments. In the visual sensing techniques, high-

1 Graduate Research Assistant, Department of Civil and Environmental Engineering, West Virginia University, Morgantown, WV 26506-6103, USA, yofeng@mix.wvu.edu

2 Assistant Professor, Department of Civil and Environmental Engineering, West Virginia University, Morgantown, WV 26506, USA, fei.dai@mail.wvu.edu

3 Assistant Research Fellow, Institute of Nuclear and New Energy Technology, Tsinghua University, Beijing, China, 100084, jqzhao@mail.tsinghua.edu.cn 
speed cameras are utilized to collect image frames of the target. Then, feature detection and matching algorithms are applied to track the spatial positon changes of the visual features detected in the images of the target. In this study, the deflection is defined as the in-plane geometric changes of structural members on the surface, which can be computed by recovering the target's spatial coordinates over time. After obtaining the deflection information, strains of the target can be calculated, which together with the deflection information will help make preventive decisions in response to different situations, for instance, sending a warning signal when the maximum strain of a civil infrastructure exceeds the pre-set threshold.

It is noticeable that since the displacement and deformation of the target are directly calculated by comparing the feature position changes, the measuring accuracy of the said techniques is primarily determined by how accurately the features can be detected and matched from the image frames of the target. Therefore, the initial and essential task for implementing the visual sensing technique to derive an accurate measuring method is to obtain the optimal feature detection and matching algorithm from a series of alternatives. In this research, we propose to compare the DIC, SIFT and SURF algorithms for the image feature detection and matching procedure. A series of experiments were designed and conducted to compare these three algorithms. In addition, the accuracy of these algorithms to measure in-plane deflections and strains was evaluated and analyzed in a detailed manner. Our experimental results revealed that the DIC algorithm has superiority among the three algorithms and holds great potential to be applied to measure in-plane deflections and strains for civil infrastructure applications.

\section{BACKGROUND}

\subsection{Methods for Measuring Deflections of Civil Infrastructures}

This section focuses on the methods that are available to measure deflections and strains for civil infrastructures, which can be categorized into contact and non-contact methods.

Contact measuring methods require the measuring instruments to be installed onto the target surface or into its body.

The first class is the wire/wireless sensor network based methods which are most typically used for measuring structural deflections. Usually professional operators install the wire/wireless sensors onto the target that needs to be monitored, whereby they can collect the target's spatial positon changing information (Kim et al. 2007). This method can achieve sufficient accuracy performance for measuring deflections. The development of wireless sensing techniques may reduce the extra expense of the wire transmission to some extent (Lynch and Loh 2006); however, the convenience of switching operations between different measuring targets still needs improvement. Besides, when facing relatively large-scale applications, the number of sensors needed for installation and uninstallation will be extra work. Moreover, since the sensor network based methods can only detect the deflections of those particular positions where the sensors are placed, the full-field deflections of the target still cannot be measured (Chintalapudi et al. 2006).

The second class is the Global Positioning System (GPS) based methods, which are in contact measuring nature since the GPS receivers need to be installed onto the surface of the target for collecting data. The GPS-based methods include static, fast-static, and RTK (real-time kinematic) modes. Previous research of GPS monitoring for civil structures includes the static monitoring of settlements and deflection trends for banks or dams (Yi et al. 2013). Also, the real-time kinematic (RTK) method has been applied in structural 
health monitoring (SHM). In the RTK mode, the target point's 3D coordinates can be determined by the static GPS receivers and by recording the difference between calculated spatial positons from the satellite data.

Non-contact measuring methods enable the measuring tools to be a few meters or even further away from the target. These methods have much more flexible spatial adaptability and less installation complexity compared to contact measuring method (such as wireless sensor networks). It actually holds more potential to be applied to measure deflections and strains for civil infrastructures when considering the operation convenience. It is particularly important when dealing with those relatively large-scale infrastructures. There are a series of methods that have been developed such as laser scanning, image/video-based methods, and total station surveying. Alba et al. (2006) proposed a method to monitor deformations of large concrete dams by terrestrial laser scanning. The advantage of this method is that it can achieve very high measuring accuracy. However, this method suffers from the high cost of a high-precision surveying laser scanner (normally, over a hundred thousand dollars), which has caused this method to not be practical for the cost-effective goal of a particular project. Maas and Hampel (2006) applied total station surveying to measure the deflections of civil infrastructures. In this method, several special markers were placed on the target to record the spatial coordinates of the markers over time in order to acquire the target's positon changing information. However, using total station faces the similar challenge as applying sensor networks. That is, it cannot achieve full-field measurement of deflections for targets.

Considering the above mentioned unfavorable factors of the discussed methods, image sensing techniques can be considered as an alternative option. They apply optical sensors (e.g., cameras) to capture and record spatial position information of targets based on relevant imaging principles (Wahbeh et al. 2003). This method has several advantages when compared to the conventional methods. First, it can generate accurate full-field deflections of the target that the field of view covers. Second, it is convenient to periodical use and applicable for different scales of civil infrastructure applications (Wang and Cuitiño 2002). As a result, the image sensing techniques can be a promising alternative to be applied to measure deflections of civil infrastructures.

The principle of the deflection measuring method using imaging lies in detecting and matching interest features in a series of continuous image frames to obtain the position changing values of the features. The accuracy of this method is primarily associated with the interest points' location in each image frame. Hence, the key task to achieve a highaccuracy measuring method is determined by performance of the feature detection and matching (Küntz et al. 2006). Therefore, obtaining the optimal feature detection, matching, and/or tracking algorithm has top priority over other tasks in our research.

\subsection{Feature Detection and Matching/Tracking Algorithms}

A plethora of algorithms have been developed to detect and match feature points cross images. These algorithms can be categorized into two types: the description-based algorithms and correlation-based algorithms. As to the description-based algorithms, we selected the SIFT algorithm (Lowe 2004) and the SURF algorithm (Bay et al. 2006) for their superiority over other detectors and descriptors (Zhu and Davari 2015). In terms of correlation-based algorithms, researchers have successfully applied them in mechanical and manufacturing engineering applications. A typical method is DIC (Zhao et al. 2012).

Lowe (2004) proposed Scale-Invariant Feature Transform (SIFT). Assessment results revealed that SIFT and its expansions have more robustness than other descriptors (Mikolajczyk and Cordelia 2005). The features extracted by SIFT are local features of the 
image. Those features have scale invariant property to spatial translation, rotation, scaling, brightness variation, occlusion, and image noise. These properties lead SIFT to be one of the most powerful algorithms for feature detection and matching in different areas.

Speeded-Up Robust Features (SURF) was proposed by Bay et al. (2006). In SURF, image features are localized by applying a non-maximum suppression schema across image scales. The Henssian matrix is used which has excellent stability when extracting local extreme points in the images. While SIFT uses the natural properties of an image without considering color, SURF makes use of the RGB information in feature extraction and matching. So far, the SURF algorithm has been applied to several applications such as automatically generating 3D points of civil infrastructures (Fathi et al. 2015).

Digital image correlation (DIC) belongs to correlation-based methods. It is an optical method that employs image registration techniques for accurate measurements of changes in images (Schreier et al. 2009). This method has been applied in applications such as industrial part deformation detection in mechanical engineering. Related research has shown the DIC algorithm has great potential in the mechanical field (Zhao et al. 2012).

\section{PROBLEM STATEMENT AND OBJECTIVE}

Although there are a plethora of algorithms available for detecting and matching feature points across images, research has revealed that SIFT and SURF detectors and descriptors have priority over other detectors and descriptors (Zhu and Davari 2015). In spite of this, the accuracy of applying them to measure structural deflections has not been assessed.

The DIC algorithm has been successfully applied in mechanical applications. However, its performance in civil engineering has not been justified. It is obvious that there are distinct differences between its applications in civil and mechanical engineering fields. For example, speckle patterns are usually used for DIC in mechanical applications (Bornert et al 2009); whereas the speckle patterns are not always appropriate to be utilized on civil infrastructures. In addition, civil infrastructures such as tunnels or dams, are usually in large scale, which may cause uncertainties of applying DIC in the field. As a result, to identify whether DIC still reveals potential to measuring deflections and strains for civil infrastructures, it is needed to test DIC with the civil infrastructure settings.

In short, all of the three algorithms (DIC, SIFT and SURF) might be applied in feature detection and matching for measuring in-plane deflections and strains of civil structures, but their performance in terms of accuracy is yet unknown. To determine which algorithm is optimal for the deflection and strain measuring task, this paper attempts to compare the accuracy of these algorithms through conducting several groups of experiments.

\section{EXPERIMENT AND RESULTS}

\subsection{Evaluating Accuracy of SIFT, SURF and DIC}

\subsubsection{Overview}

The Manta G-233-B industrial camera with a 2/3 inch COMS sensor and a $50 \mathrm{~mm}$ fixed focal length lens was employed. We captured two groups of video frames of the object under operating the servo-hydraulic fatigue testing machine (INSTRON 8501) in the structural lab at the West Virginia University. The machine has the meter to record the distance the testing table hoists which can be used as ground truth. The camera collects the data which is processed with three algorithms to result in three observations. 
Two groups of video frame dataset were collected in the experiments. The images in Group 1 were taken with 15.4 pixel deflection (equal to $2 \mathrm{~mm}$ displacement in real world) between each two consecutive images (the machine was configured to pause at each displacement of $2 \mathrm{~mm}$ ), and the images in Group 2 were taken with 22.9 pixel deflection (equal to $3 \mathrm{~mm}$ real world displacement interval; the machine was configured to pause at each displacement of $3 \mathrm{~mm}$ ). All testing images used in the experiments had a $2048 \times 1088$ resolution. The initial images shot for each group are presented in Figure 1.
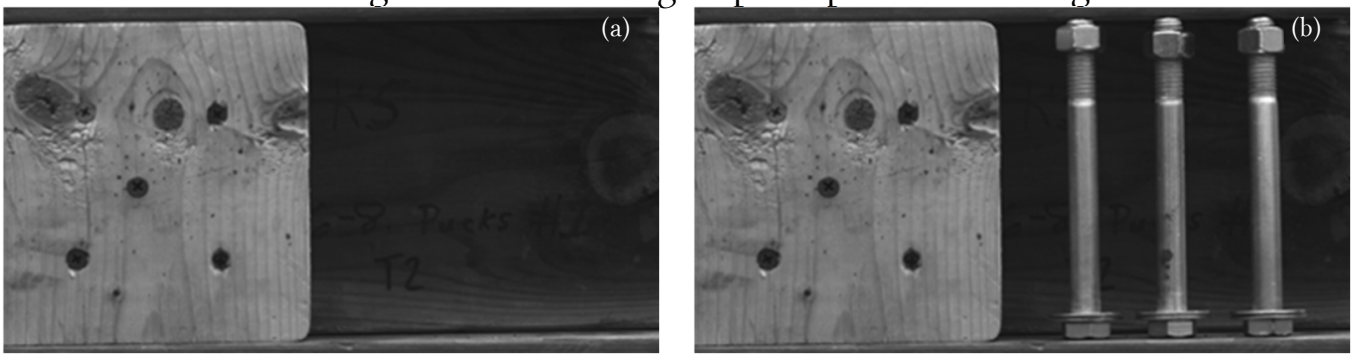

Figure 1: Sample images of (a) Group 1 and (b) Group 2

\subsubsection{Experiment Result}

The three algorithms were employed to process the two groups of images respectively. The processing results are presented in Figure 2. The calculation of the error was based on the vertical distance a feature point travels from its original position. The data collection distance was $0.95 \mathrm{~m}$. The measurement error was in the unit of pixels.

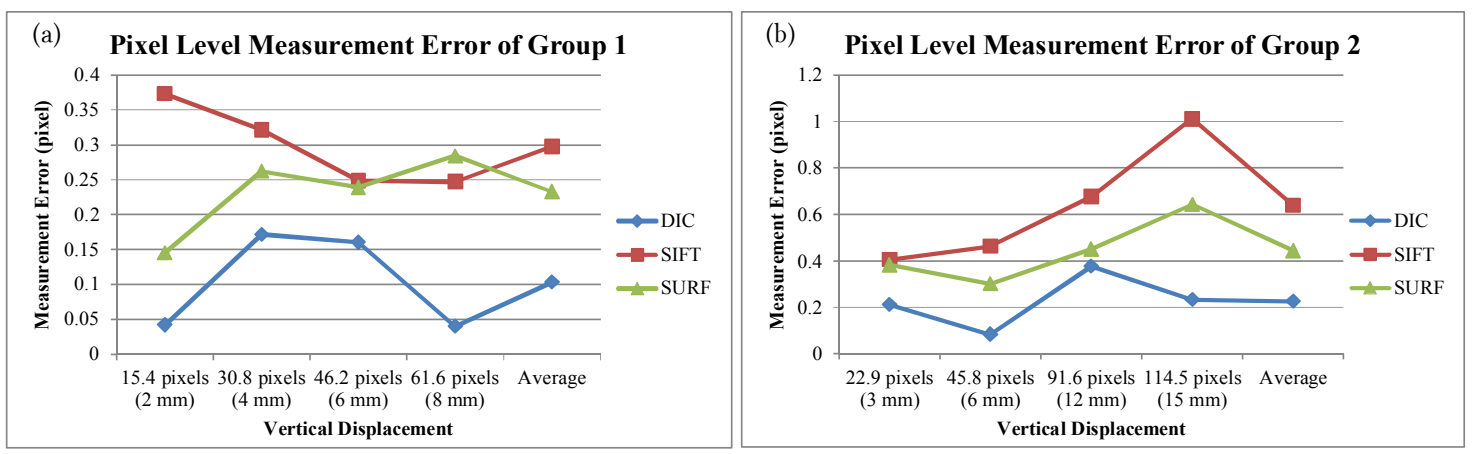

Figure 2: Pixel-level measurement error for (a) Group 1 and (b) Group 2

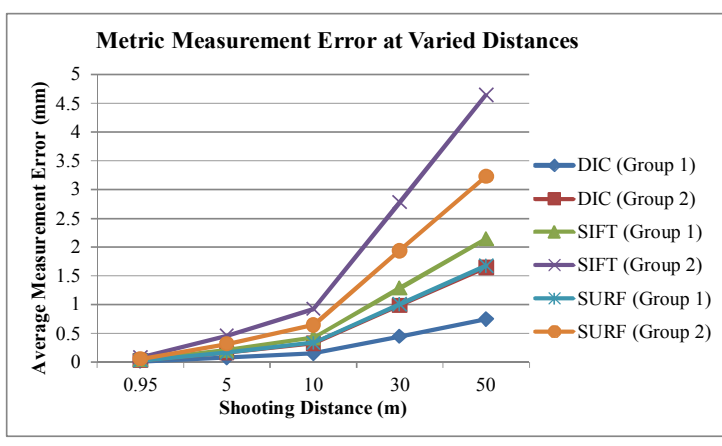

Figure 3: Metric measurement error

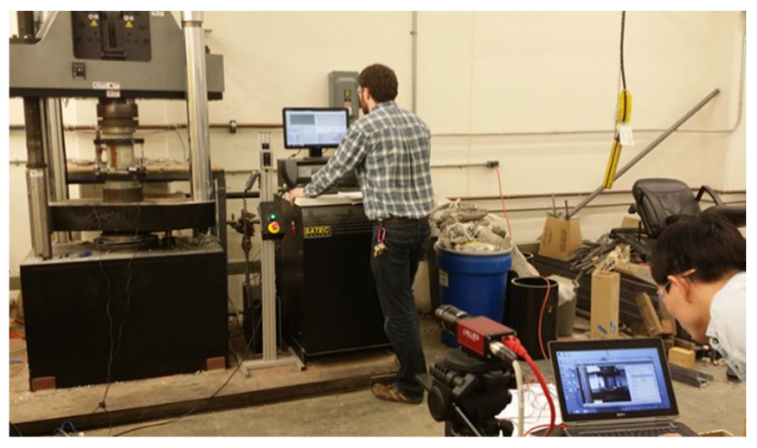

Figure 4: Experiment setting

Specifying a metric measurement of a distance in the scene makes it possible to convert pixel measurements into absolute metric values. In this experiment, the actual data collection distance of $0.95 \mathrm{~m}$ was used for unit conversation and scaling. Figure 3 presents the results of average errors for measurements at different distances. 


\subsection{Assessing Accuracy of DIC to Measuring Deflections and Strains}

\subsubsection{Overview}

Based on the results in Section 4.1, it was found that the DIC algorithm led to the optimal measuring accuracy. Therefore, in this section, we further conducted experiment to measure accuracy of applying the DIC algorithm in computing deflections and strains.

Figure 4 shows the experiment setting, in which the Manta G-233-B industrial camera with a $2 / 3$ inch COMS sensor and a $50 \mathrm{~mm}$ fixed focal length lens was used to collect video frames and two LVDT (Linear Variable Differential Transformer) instruments were applied to record the specimen movements as ground truth data. In this experiment, a concrete specimen was placed on the steel base of the INSTRON compression machine and compressed with hydraulic power supply loadings. The machine controller paused movements of the compression head at each preset distance, which was 0.0008 inches. Each time when the machine paused, the images were recorded and collected for processing. This way, the sensor measurement results could be compared with the data recorded by the machine for accuracy evaluation purpose.

Two sets of images were collected for assessments of two scenarios. As to scenario 1, the ground truth was intentionally deflected by $2 \mathrm{~mm}$ along vertical direction between each collecting frames. That was 15.4 pixels in image scope. For scenario 2, the ground truth was $3 \mathrm{~mm}$ (22.9 pixels in image scope) along vertical direction. Figure 5 presents samples of two consecutive video frames in this experiment.

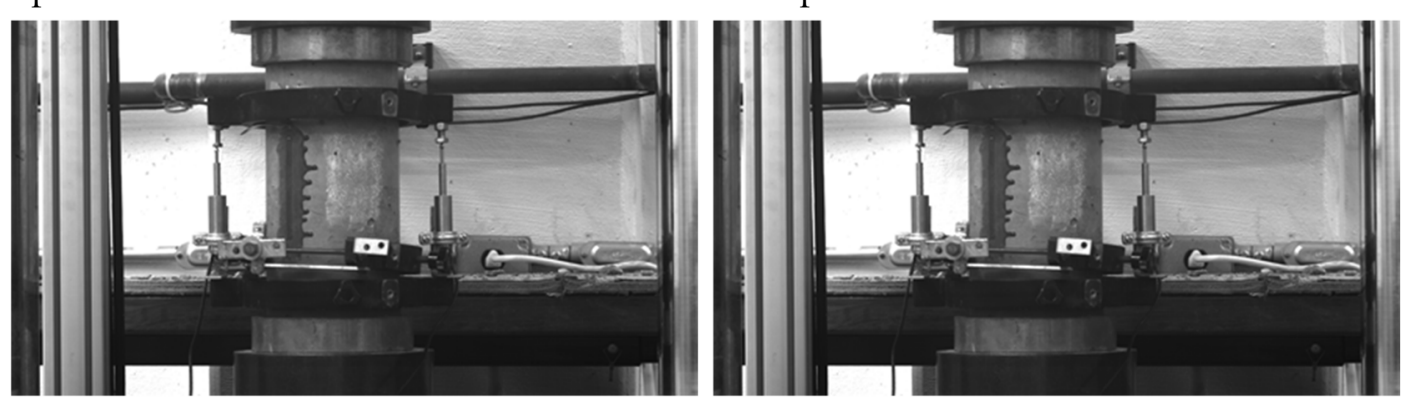

Figure 5: Samples of two consecutive video frames in this experiment

\subsubsection{Experiment Result}

In this experiment, we used the percentage error to reflect the accuracy of the algorithm. The Percentage Error could be computed by Abs (Measuring Result - Ground Truth) / Ground Truth * $100 \%$. Then, the Accuracy of the algorithm could be calculated based on $100 \%$ - Percentage Error. Tables 1 and 2 present the percentage errors for measuring deflections and strains respectively. 
Table 1: Deflection measuring accuracy

\begin{tabular}{lcccccc}
\hline & & & & \multicolumn{4}{c}{ Percentage Error (\%) } & Accuracy (\%) \\
\hline & Group 1 & Group 2 & Group 3 & Group 4 & Average & Average \\
Scenario 1 & $0.277 \%$ & $0.552 \%$ & $0.344 \%$ & $0.059 \%$ & $0.308 \%$ & $99.69 \%$ \\
Scenario 2 & $0.899 \%$ & $0.167 \%$ & $0.387 \%$ & $0.208 \%$ & $0.415 \%$ & $99.58 \%$ \\
\hline
\end{tabular}

Table 2: Strain measuring accuracy

\begin{tabular}{ccccccc}
\hline & Group 1 & Group 2 & Group 3 & Group 4 & Average & Ground truth \\
\hline Strain Value & 0.000529 & 0.000562 & 0.000555 & 0.000406 & 0.000513 & 0.000501 \\
Accuracy (\%) & $94.25 \%$ & $87.65 \%$ & $89.09 \%$ & $81.11 \%$ & $88.02 \%$ & \\
Standard Dev. & 0.000125 & 0.000101 & 0.000085 & 0.000133 & 0.000111 & \\
\hline
\end{tabular}

\subsection{Discussions}

\subsubsection{DIC, SIFT and SURF Comparison}

Based on the results (Figure 2), the DIC algorithm achieved the highest accuracy among the three algorithms. SURF was the second. In contrast, SIFT revealed the worst accuracy performance, whose average error could be as high as three times of DIC.

In terms of metric error estimation (Figure 3), DIC revealed superiority among all algorithms, SURF was the second, and the accuracy of SIFT was not comparable to DIC and SURF. For example, when the capturing distance was $50 \mathrm{~m}$, the average error of SIFT was over $4.5 \mathrm{~mm}$, which was considered meaningless for high-accuracy applications.

All three algorithms detects the natural features on the surface of the material and use them in the process of matching and tracking. It is worth noting that these visual sensing algorithms normally perform on texture rich surface. Considering that the civil infrastructure surface material typically features clutters, paints, and unsmooth finishing, all of which are of help to present distinctive visual features, it is possible to exploit the techniques for measuring deflections and deformations in civil infrastructure applications.

\subsubsection{Measuring In-Plane Deflections and Strains}

From Table 1, we could observe that the accuracy of measuring in-plane deflections was $99.69 \%$ for scenario 1 , and $99.58 \%$ for scenario 2 . The experimental results revealed that the DIC algorithm possesses the potential to be implemented for measuring in-plane deflections more than SIFT and SURF do in terms of measuring accuracy performance.

In addition, from the data shown in Table 2, the average accuracy for measuring strains of the lab scenarios was around $88.02 \%$, and the average standard deviation was $1.11 \times 10^{-}$ 4. This revealed that the DIC-based measuring method could achieve high accuracy to measure both in-plane deflections and planar strains for concrete materials.

\section{CONCLUSIONS}

This research compared the description-based SIFT, SURF algorithms and the correlationbased DIC algorithm in detecting and matching/tracking image features for measuring 
structural in-plane deflections and planar strains. We designed and conducted experiments to reveal the performance of the three algorithms. The results revealed that the DIC algorithm had much higher measuring accuracy than the SIFT and SURF algorithms. Therefore, we concluded that the DIC method has great potential to be utilized in the development of visual sensing techniques for measuring in-plane deflections and strains of civil infrastructures. However, several hurdles need to be tackled for the consideration of field implementation. For example, the efficiency of the techniques needs investigation to suffice the needs of real-time or near real-time applications. In reality, measurements in three-dimensional scope are also needed by engineers which calls for further extension of the current study. In addition, it is still challenging to apply visual sensing techniques in practices due in large part to sensitivity to the surface material (texture, color) of structures for successful applications.

\section{ACKNOWLEDGMENTS}

The authors of this paper would like to thank the NASA West Virginia Space Grant Consortium for its support of this research.

\section{REFERENCES}

Alba, M., Fregonese, L., Prandi, F., Scaioni, M., and Valgoi, P. (2006). Structural monitoring of a large dam by terrestrial laser scanning. International Archives of Photogrammetry, Remote Sensing and Spatial Information Sciences, 36(Part 5), 6.

Bay, H., Tuytelaars, T., and Van Gool, L. (2006). SURF: Speeded Up Robust Features. Lecture Notes in Computer Science, Vol. 3951, 404-417.

Bornert, M., Brémand, F., Doumalin, P., Dupré, J. C., Fazzini, M., Grédiac, M., and Wattrisse, B. (2009). Assessment of digital image correlation measurement errors: methodology and results. Experimental Mechanics, 49(3), 353-370.

Chintalapudi, K., Fu, T., Paek, J., Kothari, N., Rangwala, S., Caffrey, J., and Masri, S. (2006). Monitoring civil structures with a wireless sensor network. Internet Computing, IEEE, 10(2), 26-34.

Fathi, H., Dai, F., and Lourakis, M. (2015). Automated as-built 3D reconstruction of civil infrastructure using computer vision: achievements, opportunities, and challenges. Advanced Engineering Informatics, 29(2), 149-161.

Karbhari, V. M., and Zhao, L. (2000). Use of composites for 21st century civil infrastructure. Comput. Methods in Appl. Mech. Eng., 185(2), 433-454.

Kim, S., Pakzad, S., Culler, D., Demmel, J., Fenves, G., Glaser, S., and Turon M. (2007). Health monitoring of civil infrastructures using wireless sensor networks. The 6th International Symposium on Information Processing in Sensor Networks, 254-263.

Küntz, M., Jolin, M., Bastien, J., Perez, F., and Hild, F. (2006). Digital image correlation analysis of crack behavior in a reinforced concrete beam during a load test. Canadian Journal of Civil Engineering, 33(11), 1418-1425.

Lowe, D.G. (2004). Distinctive image features from scale-invariant keypoints. International Journal of Computer Vision, 60(2), 91-110.

Lynch, J.P., and Loh, K.J. (2006). A summary review of wireless sensors and sensor networks for structural health monitoring. Shock and Vibration Digest, 38 (2), 91-130

Maas, H. G., and Uwe, H. (2006). Photogrammetric techniques in civil engineering material testing and structure monitoring. Photogramm. Eng. Rem. S., 72(1), 39-45. 
Mikolajczyk, K., and Cordelia, S. (2005). A performance evaluation of local descriptors. Pattern Analysis and Machine Intelligence, IEEE Transactions on, 27(10), 1615-1630.

Schreier, H., Orteu, J., and Sutton, M. A. (2009). Image Correlation for Shape, Motion and Deformation Measurements - Basic Concepts, Theory and Applications, Springer.

Wahbeh, A.M., John, P.C., and Sami, F.M. (2003). A vision-based approach for the direct measurement of displacements in vibrating systems. Smart Mater. Struct., 12(5), 785.

Wang, Y., and Cuitiño, A.M. (2002). Full-field measurements of heterogeneous deformation patterns on polymeric foams using digital image correlation. International Journal of Solids and Structures, 39(13), 3777-3796.

Yi, T.H., Li, H.N., and Gu, M. (2013). Recent research and applications of GPS-based monitoring technology for high-rise structures. Struct. Control. Health Monit., 20(5), 649-670.

Zhao, J.Q., Zeng, P., Lei, L.P., and Ma, Y. (2012). Initial guess by improved populationbased intelligent algorithms for large inter-frame deformation measurement using digital image correlation. Optics and Lasers in Engineering, 50(3), 473-49.

Zhu, Z., and Davari, K. (2015). Comparison of local visual feature detectors and descriptors for the registration of 3D building scenes. J. Comput. Civ. Eng., 29(5), 04014071. 